\title{
Recommendation System for Adaptive E-learning using Semantic Net
}

\author{
V. Senthil kumaran \\ PSG College of Technology \\ Peelamedu, Coimbatore - 641004 \\ India
}

\author{
A. Sankar \\ PSG College of Technology \\ Peelamedu, Coimbatore - 641004 \\ India
}

\begin{abstract}
In the e-learning system an abundant amount of information is created and delivered to the learners over electronic media. Learners are often getting confusion by the flow of information and have difficulty in selecting the topic to learn that satisfies their needs and interests. There are several researches have been performed to provide personalized learning paths for individual learners. But many of them collect the learners' interest, habits and behavior from their profile and based on that they recommend learning path. It is the fact that the learners' interest, learning attitude and need will vary from time to time and course to course. In this paper a recommendation system is proposed using semantic net that helps the learners by offering a more intelligent approach to navigating and searching course content. In this the learner will get more personalized and contextual recommendation. The results show that semantic net based methods enable interoperability of heterogeneous course content representation and result in accurate recommendations. The validity of the proposed model is shown using sample learners and performance measures for the recommendation effects are given for evaluating the proposed system.
\end{abstract}

\section{Keywords}

Recommendation System; Semantic Net; Learner's behavior; adaptive e-learning; course filtering.

\section{INTRODUCTION}

Since there is an abundant amount of information is created and a rapid growth of information delivered to the learners in e-learning, the recommendation system for e-learning becomes an important research area. The main purpose of the recommendation system is to reduce the irrelevant content and to provide the learners with more relevant and tailored information. Hence a lot of approaches to the recommendation systems have been developed and applied to various applications [13]. Most of the research on e-learning concentrates on representing the course content and providing the content to the learners in an intelligent way [14]. However, in most of the e-learning system courses and course content are fixed one and the pedagogical methods used to select the learning path / learning objects is dynamic[15]. So, with the rapid growth of information in e-learning, it is becoming increasingly hard for learners to find the information they need. Making recommendations in elearning is different from that in other domains. The issues for an e-learning recommendation system are [16]: Items liked by learners might not be pedagogically appropriate for them and customization should not only be made about the choice of learning items, but also about their delivery. In this paper we propose a recommendation system that helps the learners by offering a more intelligent approach to navigating and searching course content. In the proposed model the recommendation is based on the course content using pedagogy, learners' preferences in the past and on the knowledge level of the learner.

A main problem in collaborative filtering systems is the availability of learners' preferences. In many applications, learners are not willing to identify themselves and the tracking of the learner behavior may be difficult. But in our system the learner's behavior is tracked by conducting test and by checking the knowledge level of the learner. The learner profiling is maintained as semantic net. The features of the content that the learner has preferred in the past can be stored in the learner's profile and used in the retrieval.

\subsection{Adaptive e-learning}

Computers are used in education a very long back. When the World Wide Web was launched in 1991, the use of computers in education has got a new era called as electronic learning (or e-learning). The use of the Web as an educational medium has created a revolution in the teaching learning process. Elearning encompasses a wide diversity of practices in a dynamic, rapidly changing field. It must therefore be defined to encompass all learning experiences involving the acquisition or transfer of knowledge. In continuation to that several researches have been performed to personalize the elearning.

A non personalized e-learning typically presents the same content to all learners regardless of the leaner's profile, their personal preferences and interest and learning attitude. Such e-learning systems experiences rely on navigation and search for learners to extract course materials. It can be argued that such e-learning systems are 'pull oriented' as it requires the learners to select an item before it is presented to them.

A personalized e-learning is in many ways the exact opposite. True - it will still have a navigation structure for learners to be able to move around the course content - and true it will still usually have a search facility for learners to 'pull' content - but the big difference is that the experience can be changed to be different to the learner based on a number of factors. It can be argued that a personalized e-learning is 'push' oriented - as content is far more controlled and can be specifically targeted at a learner rather than relying on them finding it. There are two types of personalized e-learning: prescriptive e-learning and adaptive e-learning. The prescriptive e-learning is rule based (profile matching). Based on the profile the content will be delivered. The adaptive e-learning is context based. It will analyze and predict the learner and create a learner model to 
recommend the material for learners. There are three stages in adaptive e-learning. Fig 1 shows the stages in adaptive elearning. The adaptive e-learning is an incremental approach.

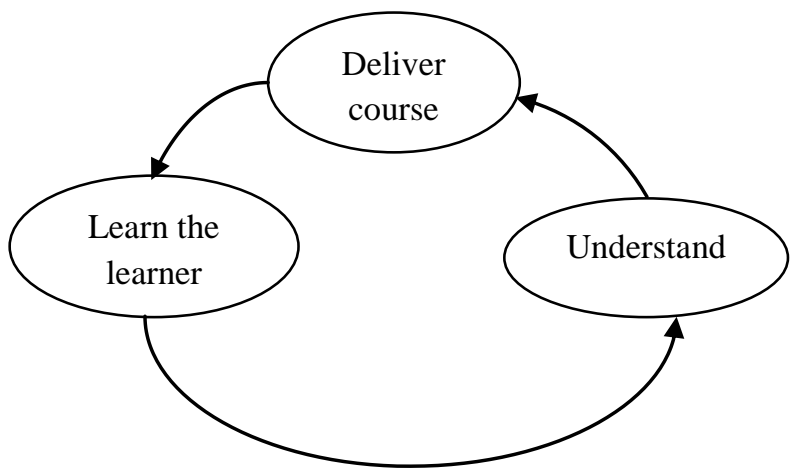

Fig 1: Stages in adaptive e-learning

\subsection{Semantic Net}

Semantic net (or Semantic network) is an alternative to predicate logic as a form of knowledge representation. The idea is that we can store our knowledge in the form of a graph, with nodes representing objects in the world, and arcs representing relationships between those objects. A semantic net (or semantic network) is a knowledge representation technique used for propositional information. So it is also called a propositional net. Semantic nets convey meaning. Semantic nets are two dimensional representations of knowledge. Mathematically a semantic net can be defined as a labeled directed graph. Semantic nets consist of nodes, links (edges) and link labels. We use semantic net to represent the course materials and learners. Each node in course material semantic net represents the topic in the course, the link represents the relationship between two topics and the link label represents the weight and ranking generated between two topics. Each node in learners semantic net represents the learners, the link represents the relationship between two learners and the link label represents the weight and ranking generated between two learners.

The rest of this paper is organized as follows. In Section 2, some related recommendation systems, e-learning systems and some related systems using semantic net are analyzed. Section 3 presents the overall architecture of the system and its working principle. In Section 4, results and discussion are given. Section 5 explores the evaluation of effectiveness of the system. We give the conclusion in section 6 .

\section{RELATED WORK}

Recommendation systems are one form of filtering information and providing the most relevant information to the user. This will provide advice to the users with the information that are likely of interest to the user [4]. Recommendation systems are most commonly used in recommending products in online stores and in departmental stores. There are several researches have been performed to provide recommendation services based on user behavior. [3] Recommendation system is an active area of research and several studies were performed to apply this in recommending books, music, movies, videos, news, web pages for searching information [5][6][7]. In the model proposed by [8] the objects are recommended based on the estimated ranks of user interest. There are four categories of recommendation system:[9][10] i) rule-based filtering, where simple rules were used ii) collaborative filtering - the users will be recommended items that people with the same tastes and preferences liked in the past.[12] iii) Content-based - the users will be recommended items that are similar to the one that the user preferred in the past. This will give poor result when all the users are heterogeneous and limited [11]. iv) hybrid system - combines both collaborative and contentbased approaches. Ontologies have been applied to a variety of recommender systems to reduce content heterogeneity and improve content retrieval [9].

An evolving web-based learning system which can adapt to the open web in response to the usage of its learning materials is proposed by Tiffany [16]. Personalization is the next step in the evolution of eLearning systems. Students can have several cognitive styles, which make the efficiency and efficacy of an eLearning system different with distinct students. Integrating the three different evolution in the area of e-learning are pedagogy (learner oriented educational theories), pragmatic (practical solutions to use of instructional components by teachers and instructional designers), and technological (building instructional components by computer professional) can be done using ontology [17]. Ontology can be used for knowledge representation. But in ontology we can specify how one topic in related with other topics and it highly impossible to include the learners view and rating. Data mining can also be used to extract the knowledge from Elearning system such as Moodle. The course recommendation system in e-learning is a system that suggests the best combination of courses in which the students are interested. Data mining techniques can be used to suggest the best combination of courses [18][19]. Some e-learning recommendation systems learn the learners' interest, attitude and need frequently, and classify them as low ability, moderate ability and high ability learners and recommend the learning path accordingly and some other learn the learner's interest, attitude and need from their profile. These systems do not worry about the suitability of the learning path to the learner. But it is the fact that the learners' interest, learning attitude and need will vary from time to time and course to course. In this paper we propose a recommendation system using semantic net that helps the learners by offering a more intelligent approach to navigating and searching course content.

\section{SYSTEM ARCHITECTURE}

The system is designed to support a leaner to get personalized recommended content. We focus on two issues for recommending suitable and relevant course material (learning objects) to the learners. First key issue in this area is how to find learner's interest, need, knowledge level and behavior effectively. Second is how to organize and recommend the course content. This paper exploits semantic net based method to overcome these issues.

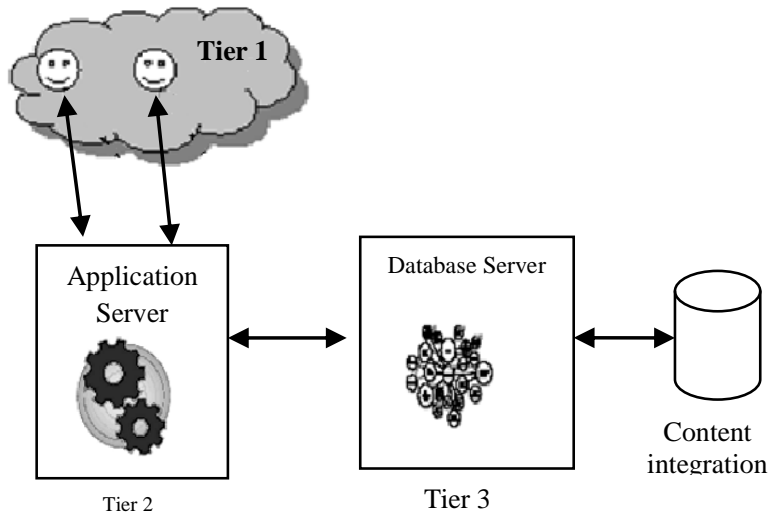

Fig 2: Recommendation System Architecture 
Recommendation process is based on two factors. Firstly, the rating of each topic by various learners is considered and a matrix is constructed. Then the learner behavior is analyzed based on the performance in the review process. The course content is well organized in various levels and relationship is maintained in semantic net. The system is decomposed as 3 tier architecture as shown in Fig 2.

The tier 1 is implemented as thin client, which could cut desktop costs. Application server maintains a registry of components and responsible for providing the services to the learners. It receives the request from the learner's machine and then recommendation system is executed. Database connectivity is made through an assembly by that way connection string is secured. The responsibilities of a recommendation system as whole is maintained in application server as i) Learner profile registration and updation ii) Performance monitoring iii) Maintains registry of components iv) Providing presentation logic v) Rating the course content vi) Recommending topics to the learners

The various components involved in Recommendation System are depicted in Fig 3 .

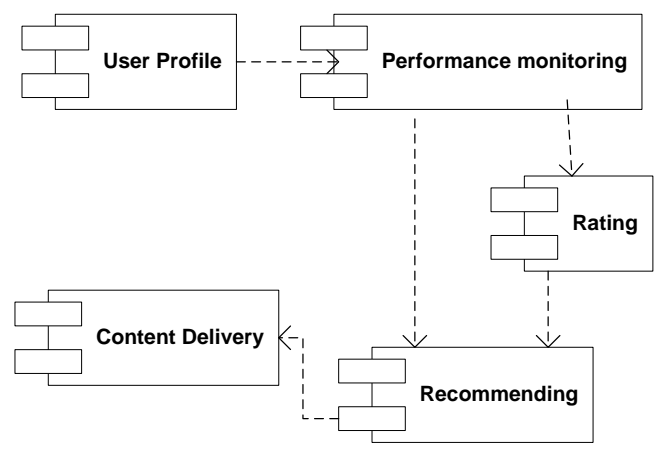

Fig 3: Component Diagram

Initially learner profile is recorded and updated continuously which enables the performance monitoring to input the recommending component. The learner will take up the course at various levels and rate (5 point scale) it after completing each level as given below.

Learner - Topic Rate Matrix for n learners L1 .. Ln and m topics $\mathrm{T} 1$.. Tm

\begin{tabular}{c|cccc} 
& $\mathrm{T} 1$ & $\mathrm{~T} 2$ & $\ldots$ & $\mathrm{Tm}$ \\
\hline L1 & 4 & 3 & $\cdots$ & 5 \\
L2 & 3 & 2 & $\ldots$. & 3 \\
$\cdot$ & & & & \\
$\cdot$ & & & & \\
$\cdot$ & & & & \\
Ln & 3 & 4 & $\ldots$. & 5
\end{tabular}

In each level of the course content, the weightage of all the links is calculated as follows:

$$
W_{t i, j}=\frac{\sum_{L 1}^{L n} R_{j}}{n}
$$

Where $W t_{i, j}$ is the weightage of link between $T i$ and $T j$, $R j$ is the rating given by the learner for the topic $T j$. This weighting factor is considered for ranking the topics and used in recommendation.

The recommending component is responsible for listing the topics as personalized content list. It is decided based on two important factors. One is rating factor and other one is with performance of the learner. If the learner's performance is good then they can take up the challenging examples and problems. By keeping this in mind recommendation component is designed.

The content delivery component is responsible for presenting the content to any type client. Initially the system checks for the type of the client from which it receives the request. Based on that the content is delivered to the learners. So that there is no restriction enforced on the client machine.

All the components are integrated for the successful implementation of recommendation system.

The course content is implemented according to the entityrelationship model for databases and is built upon the following five objects and maintained as semantic net, see Fig. 4:

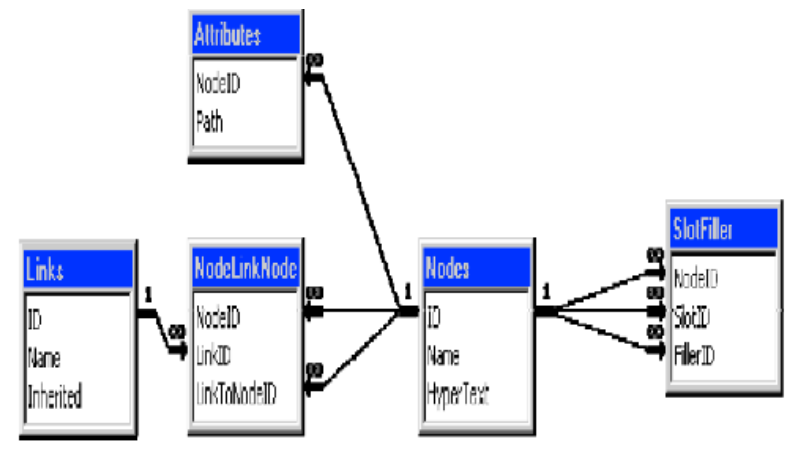

Fig 4: ER model of Course Content

- Nodes represent nodes in the semantic network; the respective attributes are identification $\langle$ ID $\rangle$, node name $<$ Name $>$ and hypertextual description $<$ HyperText $>$;

- Links stand for links in the semantic network; the attributes are identification 〈ID>, link name <Name $\rangle$ and description of inheritance <Inherited> with weightage;

- Attributes describe multimedia node structure attributes, along with identification 〈NodeID> and multimedia document paths <Path>;

- SlotFiller denotes the node triple: identification <NodeID>, identification slot <SlotID> and identification filler $<$ FillerID $>$; 
- NodeLinkNode shows connections between nodes and links in the semantic network; the respective attributes comprehend identification node <NodeID>, link $<$ LinkID> and attribute for node and link connection $<$ LinkToNodeID $>$ to store weightatge.

The formalization of learner behavior and knowledge level is based on the same syntax and semantics for nodes and links, and harmonized with knowledge representation using semantic networks. Evaluation of a learner's behavior is enabled by particularly devised point criteria. The point criteria provide quantitative and qualitative descriptions of learner activity in the learning process for the particular subject. The system eventually offers the learners a description of their success, explanations and recommendations for future work.

\section{RESULTS AND DISCUSSION}

The recommendation system for e-learning compares the collected data of a learner to similar data collected from others and calculates a list of recommended items for the learner. In recommendation system, the utility of a topic is usually represented by a rating, which indicates how a similar learner liked a particular topic. In this we use learner behavior to model and discover useful patterns of behavior.

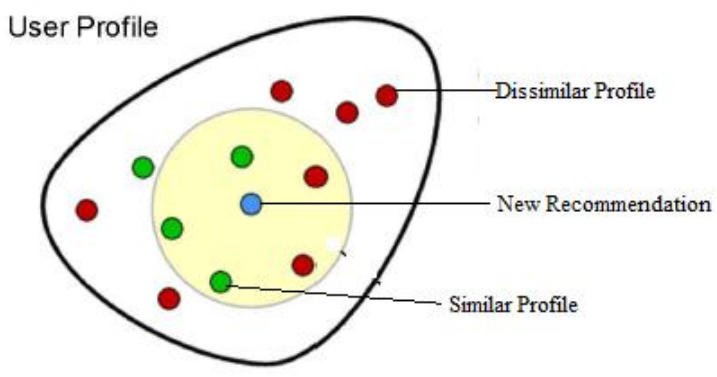

Fig 5 : Relevance of a new recommendation based on similar learner profile

The learner data is collected based on learner's log data and the result of the assessment test is also taken and analyzed the learner information and mapped to the corresponding concept in Semantic Net and prepared the recommendation list based on the learner behavior pattern. The merit of Semantic Net lies in its ability to provide a clear explained conceptual description of relationships between entities in a specific domain. Hence Semantic Net based learner modeling has been used to generate learner profile to support the development of personalized information service.

Calculated the weight based on the learner's interest conceptually and adapt relevance feedback dynamically and the same is represented in the Semantic Net so that the learner profile could reflect learner's interest. The domain concept is represented in the Semantic network.

Suppose there are 5 learners A, B, C, D, E where A, B are novice learners, $\mathrm{C}, \mathrm{D}$ are Advanced Learners and $\mathrm{E}$ is medium level. They all log on to the system at difference time to learn "Operating Systems" subject. The system generates a set of recommendations based on the behavior of the learner. The learner's behavior is stored in the learner profile to further process these recommendations. Based on the selection made by the learner from the set of recommendation, the system scores the new recommendation according to how similar it is to the other learners stored in the learner's profile (Fig. 5). This score measures the relevance of a new recommendation to the learner.

Table 1: Sample recommendation based on learner profile

\begin{tabular}{|l|l|l|}
\hline Learners & $\begin{array}{l}\text { Learner } \\
\text { Behavior }\end{array}$ & $\begin{array}{c}\text { Recommended topic based on } \\
\text { similar learner profile }\end{array}$ \\
\hline A, B & Novice & Fundamentals of Computer \\
\hline C, D & $\begin{array}{l}\text { Advance } \\
\text { Learner }\end{array}$ & Operating Systems - Overview \\
\hline E & Medium & $\begin{array}{l}\text { Introduction to Operating } \\
\text { Systems }\end{array}$ \\
\hline
\end{tabular}

\section{EVALUATION OF SYSTEM EFFECTIVENESS}

The evaluation methodology adopted in this study is similar to the one described in [20]. For the dataset, known weights are split into two subsets: training set $\mathrm{M}$ and test set $\mathrm{T}$. The test set $\mathrm{T}$ contains only weights more than $90 \%$. So we can reasonably state that $\mathrm{T}$ contains items relevant to the respective learner. We adopted a procedure for our dataset. We randomly collect $1.4 \%$ of the weights range from the dataset in order to create a probe set. The training set $\mathrm{M}$ contains the remaining weights. The test set $\mathrm{T}$ contains all the weights more than $90 \%$ from the probe set. In order to measure recall and precision, we first train the model over the weights in $\mathrm{M}$. Then, for each item i with weight more than $90 \%$ by user $\mathrm{u}$ in $\mathrm{T}$ :

(i) Randomly selected 1000 additional items unweighed by user $\mathrm{u}$. Assume that most of them will not be of interest to user $\mathrm{u}$.

(ii) Predicted the weights for the test item i and for the additional 1000 items.

(iii) Formed a ranked list by ordering all the 1001 items according to their predicted weights. Let $p$ denote the rank of the test item i within this list. The best result corresponds to the case where the test item $\mathrm{i}$ precedes all the random items (i.e., $\mathrm{p}=1$ ).

(iv) Formed a top-N recommendation list by picking the $\mathrm{N}$ top ranked items from the list. If $\mathrm{p} \leq \mathrm{N}$ we have a hit (i.e., the test item $\mathrm{i}$ is recommended to the user). Otherwise we have a miss. Chances of hit increase with $\mathrm{N}$. When $\mathrm{N}=1001$ we always have a hit.

In the e-learning environment the relevant topic returned through the recommendation system will be the primary basis to determine the quality of the system. Precision and recall are the two major indices of information retrieval. Precision expresses the proportion of relevant topic among the topics recommended, while recall expresses the proportion of topic recommended from the relevant topics. The formulae are, respectively outlined below:

$$
\begin{aligned}
& \text { Pr ecision }=\frac{A}{A+B} \\
& \operatorname{Re} \text { call }=\frac{A}{A+C}
\end{aligned}
$$


Where A is the relevant topic recommended, B presents the irrelevant topic retrieved by the system and $\mathrm{C}$ is relevant topic that the system not recommended. Here the precision will be used in evaluating the proportion of learner interests towards the topics stored in the system. And recall estimates the ability that the system can learn and also examines the degree that the system is able to satisfy the needs of learners. The precision and recall value are computed by uploading course topics for the subject "Operating Systems" and by using 10 learners of different behavior level and is shown in Fig 6.

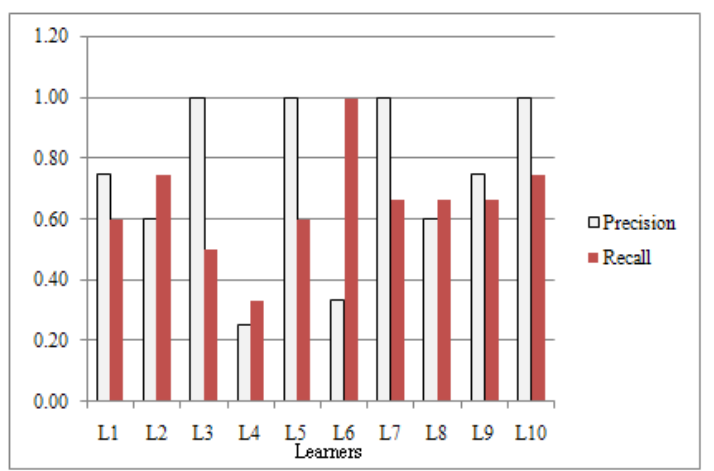

Fig 6: Chart showing the precision and recall for 10 learners

Table 2: Average Relevant and Retrieved recommendation

\begin{tabular}{|c|c|c|}
\hline & Relevant & Irrelevant \\
\hline Retrieved & 3 & 1 \\
\hline Not Retrieved & 2 & 4 \\
\hline
\end{tabular}

Table 2 shows the number of relevant and irrelevant documents retrieved and not retrieved. The evaluation measure values are average precision 0.828 and Recall 0.653 . These measures show that the recommendations made by the system are very relevant to the learner.

\section{CONCLUSION}

The analysis of the impact of recommendation in adaptive elearning on student performance with questions of different complexity (strong, moderate, low) leads to some interesting observations. First, it seems that recommendation in adaptive e-learning encourages students to do more work and creates interest among the students. Second, once if they understand the fundamentals with easy questions, it pays back across all three complexity levels. In this paper, a semantic network based approach to recommendation system for e-learning is presented. This research work involves about using both the learner's behavior and service to learners to recommend topics to study. It generates the learner profile and creates the semantic network for learner and then creates the domain semantic network. The learner's interest is discovered and recommendations are made accordingly. As a style of recommendation, use of learner's behavior and learner's learning style makes particular sense in the context of personalized learning. It is proved that semantic net-based methods enable interoperability of heterogeneous course content representation and result in accurate recommendations.

\section{References}

[1] Tuukka Ruotsalo, Lora Aroyo, and Guus Schreiber, (2009) "Knowledge-Based Linguistic Annotation of Digital Cultural Heritage Collections" IEEE Intelligent Systems, 24(2), pp. 64-75.

[2] Tuukka Ruotsalo, Eetu Mäkelä, Tomi Kauppinen, Eero Hyvönen, Krister Haav, Ville Rantala,Matias Frosterus, Nima Dokoohaki, and MihhailMatskin, (2009) "Smartmuseum: Personalized Context-aware Access to Digital Cultural Heritage", In Proceedings of the International Conference on Digital Libraries and the Semantic Web 2009 (ICSD 2009), September, pp. 178192. Università diTrento, Trento, Italy.

[3] Tomoharu lwata, Kazumi, Takeshi Yamada, (2007) "Modelling User Behavior in Recommender Systems based on Maximum Entropy", Proceedings of the 16th international conference on World Wide Web, pp. 1281 1282

[4] Robin Burke, (2000) "Knowledge-based Recommender Systems", In: A. Kent, Encyclopedia of Library and Information Systems, 69(32).

[5] Greg Linden, Brent Smith, and Jeremy York, (2003) "Amazon.com recommendations item-to-item collaborative filtering”, IEEE Internet Computing, 7(1), pp. 76-80.

[6] Joseph A. Konstan, Bradley N. Miller, David Maltz, Jonathan L. Herlocker, Lee R. Gordon, and John Riedl, (1997) "GroupLens: applying collaborative filtering to Usenet news", Communications of the ACM , 40(3), pp.77-87.

[7] Bradley N. Miller, Istvan Albert, Shyong K. Lam, Joseph A. Konstan, and John Riedl, (2003) "MovieLens unplugged: experiences with an occasionally connected recommender system", In: IUI '03: Proceedings of the 8th International Conference on Intelligent User Interfaces, pp. 263-266. ACM, New York, NY, USA.

[8] Gediminas Adomavicius and Alexander Tuzhilin, (2005) "Toward the next generation of recommender systems: a survey of the state-of-the-art and possible extensions", IEEE Transactions on Knowledge and Data Engineering, 17(6), pp. 734-749.

[9] Qi Gao, junwei YAN, MMin LIU, (2008) "A Semantic approach to Recommendation System based on user ontology and spreading activation model", In the proceedings of the International Conference on Network and Parallel Computing (IEEE), pp. 488 - 492.

[10] T.P. Liang, (2007) "A semantic expansion approach to personalized knowledge recommendation", Decision Support Systems, 45(3), 2007

[11] Raymond J.Mooney and Loriene Roy, (2000) "Contentbased book recommending using learning for text categorization', In: DL '00: Proceedings of the fifth ACM conference on Digital libraries, pp 195-204. ACM, New York, NY, USA.

[12] David Goldberg, David Nichols, Brian M. Oki, and Douglas Terry, (1992) "Using collaborative filtering to weave an information tapestry", Communications of the ACM, 35(12), pp. 61-70.

[13] Gediminas Adomavicius and Alexander Tuzhilin, (2005) "Toward the next generation of recommender systems: a 
survey of the state-of-the-art and possible extensions", IEEE Transactions on Knowledge and Data Engineering, 17(6), pp. 734-749.

[14] Javed Khan and Manas Hardas, (2007) "Hierarchical Course knowledge representation using ontologies", In: IICAI'07: Proceedings of the 3rd Indian International Conference on Artificial Intelligence, pp. 1684 - 1698.

[15] Adriana da Silva Jacinto, (2008) "An ontology-based architecture for intelligent tutoring system", Interdisciplinary Studies in Computer Science. 19 (1) pp. $25-35$

[16] Tiffany Ya TANG, Gordon Mccalla (2005) "Smart Recommendation for an Evolving E-learning System: Architecture and Experiment", International Journal on e-Learning, 4(1),pp. 105-129 .
[17] H. Srimathi, (2010) "Knowledge Representation of LMS using Ontology", International Journal of Computer Applications, 6(3), pp. $35-38$.

[18] Sunita B. Aher, Lobo L.M.R.J., (2012) "Mining Association Rule in Classified Data for Course Recommender System in E-learning", International Journal of Computer Applications, 39(7), pp. 1 - 7.

[19] Sunita B. Aher, Lobo L.M.R.J., (2011) "A Framework for Recommendation of courses in E-learning System", International Journal of Computer Applications, 35(4), pp. $21-28$

[20] Y. Koren, (2008) "Factorization meets the neighborhood: a multifaceted collaborative filtering model", In KDD '08: Proceeding of the 14th ACM SIGKDD international conference on Knowledge discovery and data mining, pp. 426-434, New York, NY, USA, 2008. ACM. 